EPJ Web of Conferences 114,02006 (2016)

DOI: $10.1051 /$ epjconf/201611402006

C Owned by the authors, published by EDP Sciences, 2016

\title{
Jet flow issuing from an axisymmetric pipe-cavity-orifice nozzle
}

\author{
Zuzana Broučková ${ }^{1, a}$, Pavlína Puškováa $^{2}$, Zdeněk Trávníček $^{1}$ and Pavel Šafařík ${ }^{2}$ \\ ${ }^{1}$ Institute of Thermomechanics, the Czech Academy of Sciences, 18200 Prague, Czech Republic \\ ${ }^{2}$ Czech Technical University, Faculty of Mechanical Engineering, 16607 Prague, Czech Republic
}

\begin{abstract}
An axisymmetric air jet flow is experimentally investigated under passive flow control. The jet issues from a pipe of the inner diameter and length of $10 \mathrm{~mm}$ and $150 \mathrm{~mm}$ which is equipped with an axisymmetric cavity at the pipe end. The cavity operates as a resonator creating self-sustained acoustic excitations of the jet flow. A mechanism of excitations is rather complex - in comparison with a common Helmholtz resonator. The experiments were performed using flow visualization, microphone measurements and time-mean velocity measurements by the Pitot probe. The power spectral density (PSD) and the sound pressure level (SPL) were evaluated from microphone measurements. The jet Reynolds number ranged $R e=$ 1600-18 000. Distinguishable peaks in PSD indicated a function of the resonator. Because the most effective acoustic response was found at higher $R e$, a majority of experiments focused on higher $R e$ regime. The results demonstrate effects of the passive control on the jet behavior. Fluid mixing and velocity decay along the axis is intensified. It causes shortening of the jet transition region. On the other hand, an inverse proportionality of the velocity decay $(u \sim 1 / x)$ in the fully developed region is not changed. The momentum and kinetic energy fluxes decrease more intensively in the controlled jets in comparison with common jets.
\end{abstract}

\section{Introduction}

A jet flow is a type of fluid motion and one of basic types of shear flows. The jet flow is very important for theoretical studies in fluid mechanics - see Schlichting [1], Abramovich [2]. On the other hand, the jet flow is very important also from practical engineering point of view because it is frequently used in industrial applications such as fluid mixing, control of flow and thermal fields, thrust generation, convective heat transfer, and combustion and chemical processes.

This study is focused on turbulent submerged continuous jets. Despite the continuous (steady) jet's capability is unquestionable, unsteadiness effects can enhance applicability even more. It is worth noting here that unsteadiness is not a foreign feature to the continuous (steady) jets, quite the contrary, a tendency to form natural organized structures and oscillations are intrinsically incorporated in all jet flows - see, e.g., Crow and Champagne [3].

There are many ways how to generate/promote a pulsatile character of a jet flow. Generally, passive and active flow control methods can be distinguished - see Gad-El-Hak [4]. Relevant to this study are the passive methods which are based on geometry modifications and which can generate large vortex structures and/or significant acoustic excitations of the main flow. The most significant advantage of the passive control methods

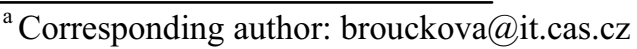

is a relative simplicity because they require neither actuators/transducers nor an external supply of energy.

A sensitivity of the jets to excitations is determined by instability and development processes either in the jet shear layers or in the entire jet columns, which are linked with the shear-layer and jet-column modes of the jet e.g. Crow and Champagne [3] and Thomas [5]. The highest sensitivity of round jets to excitation, i.e. the most amplified mode under small artificial external excitations, is known to be at the most preferred mode linked with the Strouhal number of 0.3-0.35 - see Crow and Champagne [3] and Vlasov and Ginevski [6]. However, the Strouhal number of the jet-column mode of round jets themselves (natural passage frequency of vortices without any artificial excitation) can be higher, e.g. the value of about 0.5 concluded Crow and Champagne [3] and 0.61 was found by Liu and Sullivan [7]. Moreover, much wider range from 0.25 to 0.85 is considered by Thomas [5], who explained the reason of this wide range as an influence of the thickness of the boundary layer at the nozzle exit.

To create large acoustic excitations of jets by means of the passive control methods, Hill and Greene [8] designed the so called "whistler nozzle" consisting of an overhanging sleeve, which was co-axially mounted at the pipe exit. They concluded that the self-sustained acoustic oscillations can effectively enhance fluid mixing, which can be useful for various application such as design of ejectors, improvements in the flow beneath V/STOL 
aircrafts in ground effect including reduction of ground heating/erosion effects, and on/off suppression of exhaust plume detectability by reduction of infrared footprints of combat aircrafts. Note that the experiments [8] were made with the pipe nozzle of a diameter $25.4 \mathrm{~mm}$ at the jet exit velocity of $54 \mathrm{~m} / \mathrm{s}$, i.e. the Reynolds number was approximately $9 \times 10^{4}$. Hasan and Hussain [9] experimentally investigated the "whistler nozzle" phenomenon as an implication of a self-excited organpipe resonance in either the full-wave mode or the halfwave mode. In a follow-up paper, Hussain and Hasan [10] proposed more complex explanation showing that the whistler excitation results from the coupling of two independent resonance mechanisms, namely the organpipe resonance and the shear-layer tone resulting from an impingement of a pipe-exit shear layer onto the collar lip. Experiments in both studies [9, 10] were made with the pipe nozzles of diameters $25.4 \mathrm{~mm}$ and $7.62 \mathrm{~mm}$ at the jet exit velocity of 36 and $60 \mathrm{~m} / \mathrm{s}$, corresponding to the Reynolds number range from $6.2 \times 10^{4}$ to $3.1 \times 10^{5}$.

The so called orifice-cavity-orifice nozzle was proposed for generation of the self-sustained acoustic oscillations by Abell and Luxton [11]. As was described by Lee [12], fluid passes successively a pipe, orifice, resonance cavity and finally issues through the second, outlet orifice. The aim was to enhance mixing in combustion applications. As was explained by Nathan [13], the orifice-cavity-orifice nozzle can enhance stability and power of flames, but it is not easy to apply it in industry because flow rate variations can cause an abrupt drop-out effect from (rather narrow) resonance regime resulting in flame blowing off. To overcome this fact and keep advantages resulting from self-sustained oscillations (such as mixing intensification, flame stability increase, and NOx emissions decrease), another method based on fluidic rather than acoustic mechanism was developed by Nathan [13], Nathan and Luxton [14] and Nathan et al. [15]. Namely, they used self-sustained precession of entire jet flow column inside a cavity.

Page et al. [16] investigated a utilization of the "whistler nozzle" phenomenon for an enhancement of a heat transfer from impinging jets. The velocity and Reynolds number were $90-153 \mathrm{~m} / \mathrm{s}$ and $6 \times 10^{4}-1.5 \times 10^{5}$, respectively.

Other methods capable to generate jet pulsations use various no-moving-part fluidic elements to excite continuous fluid supply. For example, flapping jets based on fluidics can be designed either with external feedback loop (Viets [17], Raman et al. [18], Camci and Herr [19], Seifert and Pastuer [20], Tesař et al. [21]) or with an internal feedback action (Mi et al., [22]).

A kettle whistle consisting of two axially aligned orifice plates (i.e. the orifice-cavity-orifice nozzle as named by Abell and Luxton [11] and cited by Lee [12]) was investigated by Henrywood and Agarwal [23]. Their microphone experiments focused on aeroacoustics. Depending on the Reynolds number $R e=U D / v$ (where $U$ is the average velocity through the exit orifice, $D$ is the exit orifice diameter, and $v$ is the kinematic viscosity), two regimes were identified. The first regime is the constant frequency regime at low Reynolds numbers
$(\operatorname{Re}<2000)$. Behavior can be modeled by the Helmholtz resonator mechanism. Instead of fundamental zero-flow case, the resonator effective neck length is a function of the flow Mach number. The second regime is the constant Strouhal number regime at higher Reynolds numbers $(\operatorname{Re}>2000)$. The driven mechanism is a vortex shedding at the exit orifice of the whistle.

As written above, Henrywood and Agarwal [23] focused on aeroacoustics behavior of the orifice-cavityorifice nozzle. However, flow field behavior of that case was not investigated neither in study [23] nor in available literature. A motivation of this study is an evident lack of information related to the flow field behavior downstream of this type of whistler nozzles. The aims of this study are to design a whistler nozzle and to demonstrate its behavior. Namely, abilities to generate self-sustained acoustic oscillations and modify the timemean jet-flow characteristics are investigated experimentally.

\section{Experimental setup and methods}

Figure 1(a) shows a schematic view of the present experimental setup. The airflow from the building compressor pass through a flexible plastic tube, ball valve (1), pressure regulator (2), throttle valve (3), passing the manometer (4) to the rotameter (5). In the case of flow visualization experiments, the airflow continues to the water fog generator (6); for velocity measurements this generator is not used. Thereafter the airflow continues through a T-shaped connecting tube (7) via the settling chamber (8) with a diameter/length of $25 / 117 \mathrm{~mm}$. The settling chamber is equipped with a honeycomb to straighten flow and suppress a swirling component of the velocity. A size of cells is approximately $4.5 \mathrm{~mm}$ and their length is $27 \mathrm{~mm}$. Thereafter the airflow continues to the investigated pipe-cavity-orifice nozzle (9), which is fixed on a traverse system (10) positioned manually along the horizontal axis; an accuracy of the adjustment is $0.1 \mathrm{~mm}$.

Figure 1(b) shows the investigated nozzle in a detail including the used cylindrical coordinate system $x, r$. The nozzle is situated vertically and it consists of an duralumin pipe of $D=10.0 \mathrm{~mm}$ internal diameter. The pipe is 15 diameters long and a perpendicular cut at the end is machined making the sharp-edged nozzle lip. The end part is equipped with a resonant cylindrical cavity with an internal diameter of $45.6 \mathrm{~mm}$. The cavity top is made out of an duralumin plate of thickens $0.4 \mathrm{~mm}$ with a sharp-edged hole of diameter $D=10.0 \mathrm{~mm}$. The depth of the cavity $h$ is can be adjusted in a range from $h=0$ (i.e. the case without the cavity) to $h=40 \mathrm{~mm}$. A more detailed description of the geometry was described by Pušková [24].

The volume flux through the apparatus thus the average velocity issuing from the nozzle are evaluated from measurements by rotameter (5) - see figure 1(a), with consideration of the pressure at the manometer (4). The rotameter is a multiple tube type with $150 \mathrm{~mm}$ tubes and built-in needle valves (Aalborg P41A6-BD1). An accuracy is $\pm 2 \%$ of full scale. The manometer is an 
electronic type with a resolution of $0.1 \mathrm{kPa}$ (Greisinger GMH 3156 with GMSD 2 BR transducer). A thermistor probe is used to measure the air flow temperature.
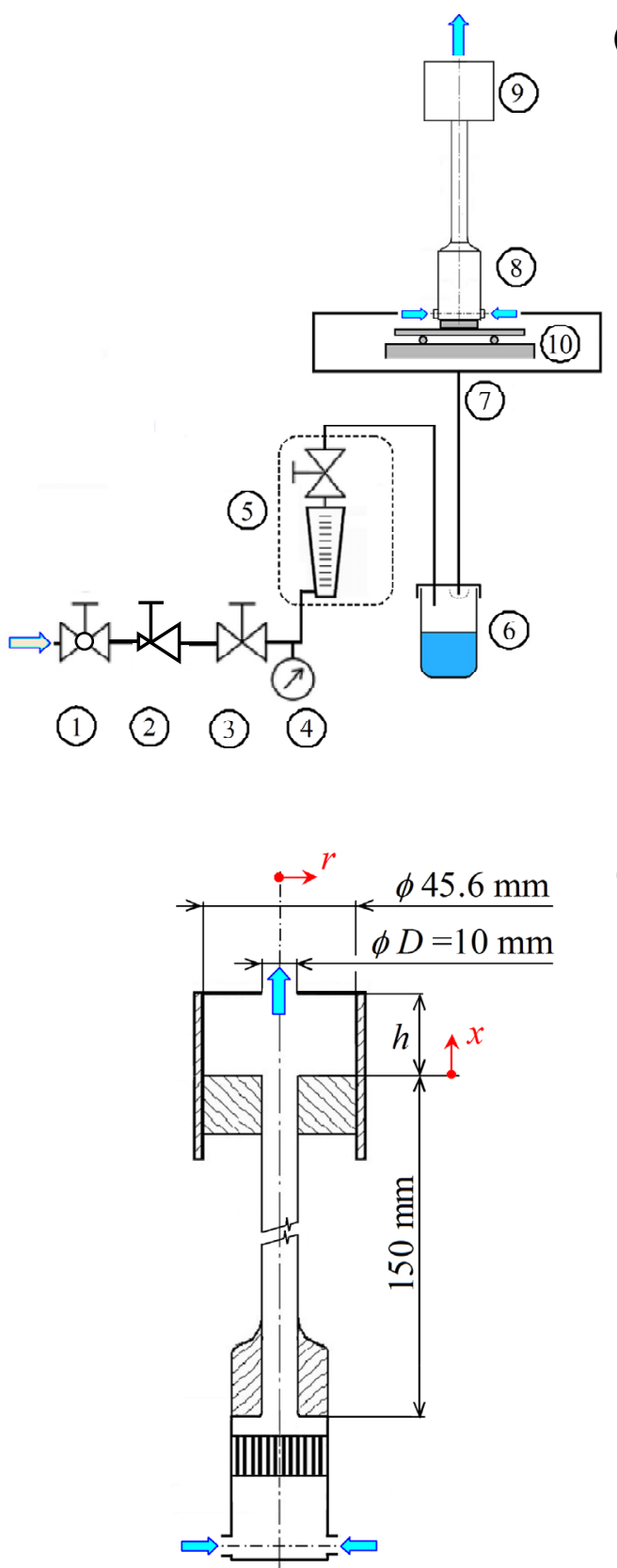

(b)

Figure 1. Experimental setup, 1: ball valve, 2: pressure regulator, 3: throttle valve, 4: manometer, 5: rotameter, 6: water fog generator, 7: T-shaped connecting tube, 8: settling chamber, 9: investigated pipe-cavity-orifice nozzle, 10: horizontal traverse system; (a) general scheme and (b) detail of the investigated nozzle.

Flow visualization used cold water fog produced by an ultrasonic piezoelectric nebulizer Mini Nebler - see figure 1(a), position 6 . Contrasting white flow field pattern (streaklines, diffused by the flow turbulence) on black background were illuminated by a (left-hand) side photoflash, located at a distance of $0.8 \mathrm{~m}$ from the nozzle axis. The photoflash was equipped by an inhouse-built lens concentrating light into about $30 \mathrm{~mm}$ thick layer along the jet axis. Pictures were taken by a digital camera which was located in front of the nozzle, at a distance of $0.8 \mathrm{~m}$ from the nozzle axis. To obtain on-line control of the camera, it was connected to a PC via a USB cable. An exposure time of $1 / 125 \mathrm{~s}$ was used.

Frequencies of the sound effects were evaluated using an electret microphone of diameter $10 \mathrm{~mm}$. The microphone was fixed perpendicularly to the nozzle axis in the place about $x=0, r=0.6 \mathrm{~m}$ and its output was connected to the sound card of a computer. The microphone signal (without calibration) was on-line analyzed by means of Spectrum Lab V2.77 software to acquire the relative amplitude of the power spectral density (PSD).

The sound pressure level (SPL) was measured by means of the Sound Level Meter QUEST 2100 with a microphone of diameter $13.5 \mathrm{~mm}$ and accuracy of $+/-0.7$ $\mathrm{dB}$. The device operates at the Frequency Weighting Networks A, which emulates the low level response of the human ear. The microphone was located close to the above mentioned electret microphone.

The time-mean flow velocity was evaluated by a Pitot tube (blunt-nosed impact tube with an outer diameter of $0.8 \mathrm{~mm})$, connected to an electronic manometer (Greisinger GMH 3156) with the transducers GMSD $25 \mathrm{MR}$ (resolution of $1 \mathrm{~Pa}$ ) and GMSD 2,5 MR (resolution of $0.1 \mathrm{~Pa}$ ). To evaluate time-mean velocity profiles, the Pitot tube probe was fixed on the traverse system positioned manually along the vertical axis. The accuracy of the adjustment was $0.5 \mathrm{~mm}$.

Considering that the static pressure is equal to the barometric pressure, the measured time-mean total overpressure $p$ can be converted into the time mean velocity as $U=c(2 p / \rho)^{0.5}$, where $c$ is the calibration factor (approximately $c=1.00$ for the present probe) and $\rho$ is the air density. An uncertainty analysis was performed according to the method of Kline and McClintock [25] for a single sample experiment. A typical centerline velocity magnitude in this study for $x=0-20 D$ was above $10 \mathrm{~m} / \mathrm{s}$. For this magnitude, the uncertainty was evaluated within $7 \%$, based on the $95 \%$ confidence level (i.e., \pm 2 standard deviation).

\section{Results and discussion}

\subsection{Helmholtz resonator}

To demonstrate possibility of the present measurements and validity of data processing, auxiliary experiments focusing on the cavity resonant frequency were performed. For this purpose, the cavity of the nozzle was adapted and the orifice from the pipe to the cavity was closed by an adhesive tape. To produce an acoustic response, an auxiliary excitation by air jets impinging obliquely onto the orifice lip $(D=10 \mathrm{~mm}$, see figure 1(b)) was used. Note that the frequency response did not depend neither on a velocity of the excitation jet nor on details of the excitation arrangement.

Figure 2 presents examples of acoustic responses as the relative amplitude of the power spectral density (PSD). Figure 2(a) is a benchmark presenting a 
background noise in the laboratory before the test - no PSD peaks are identifiable.
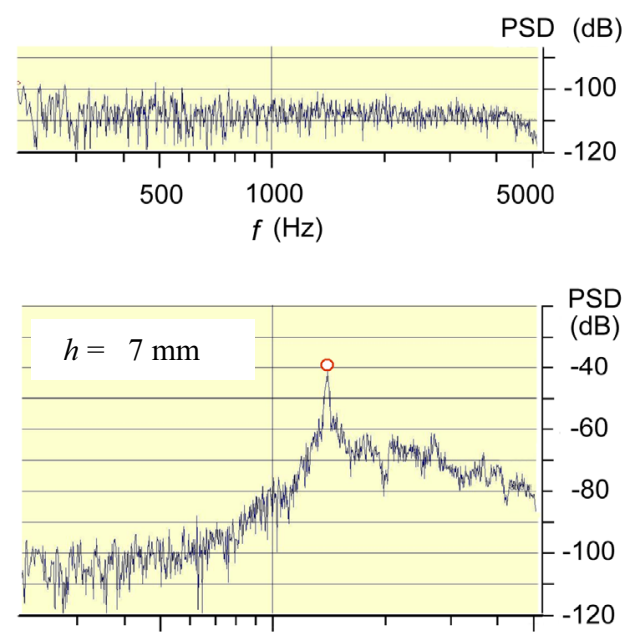

(b)
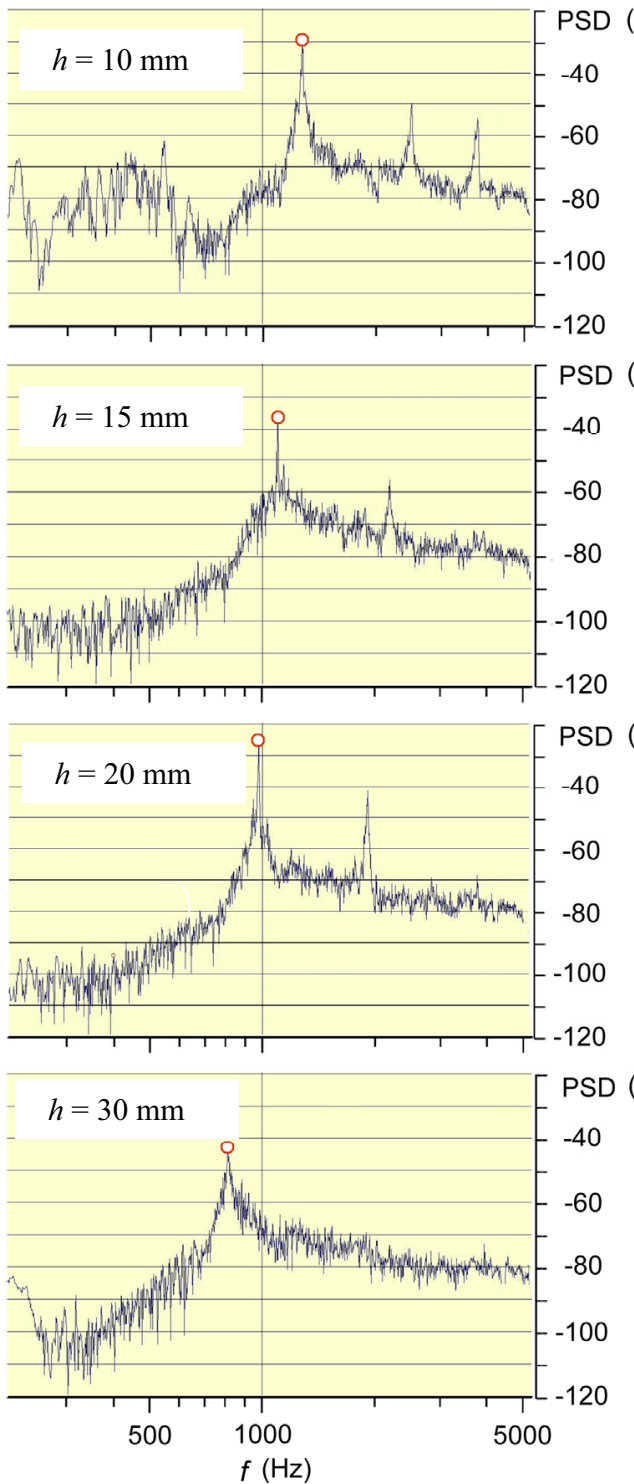

Figure 2. Power spectral density, (a) background noise, (b) acoustic response of the cavity for $h=7-30 \mathrm{~mm}$.

Sequentially, excitation air jets was used and figure 2(b) shows an acoustic response for various cavity

(a) volumes expressed as cavity depths of $h=7-30 \mathrm{~mm}$. A distinguishable PSD peaks clearly indicate the resonant

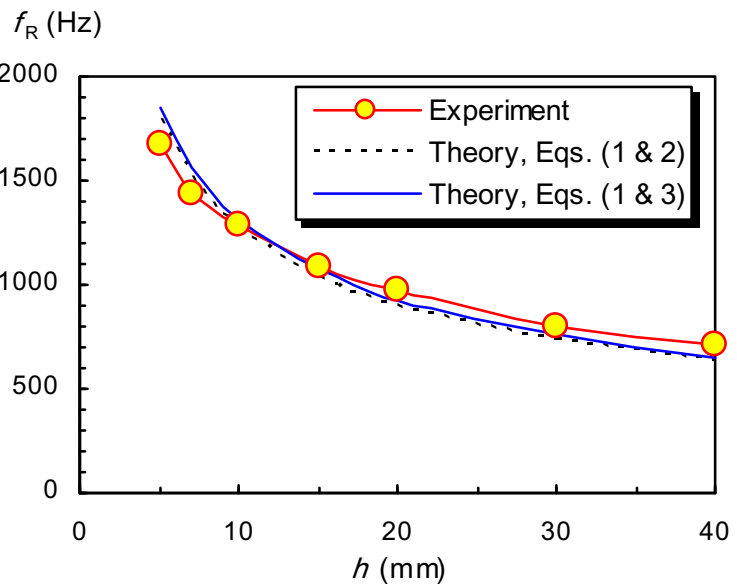

Figure 3. Resonant frequency - comparison of the present experimental data with theory.

frequencies $f_{\mathrm{R}}$. Moreover, higher harmonics are manifested by other peaks with lower PSD level, see e.g. graph for $h=10 \mathrm{~mm}$. As was expected (Kinsler [26]), an increase of the cavity volume, thus a depth increase in figure 2(b), causes a decrease of the resonant frequency. The present experimental data are plotted as a relationship between the resonant frequency $f_{\mathrm{R}}$ an the cavity depth $h$ in figure 3 . The result is well comparable with the natural frequency of the Helmholtz resonator (Kinsler [26]), which can be derived, considering the lumped acoustic system, isentropic expansion, ideal gas law, and compression during pressure oscillations, as

$$
f_{\mathrm{R}}=\frac{c}{2 \pi} \sqrt{\frac{A}{L_{\mathrm{eff}} V}},
$$

where $c$ is the speed of sound, $A$ is the cross-sectional area of the orifice $\left(A=\pi D^{2} / 4\right), V$ is the cavity volume ( $V=A h)$, and $L_{\text {eff }}$ is the effective length capturing mass of an oscillated fluid column (i.e., the slug flow model is assumed). Considering end correction for a flanged orifice (Kinsler [26]), the $L_{\text {eff }}$ can be approximated as

$$
L_{\text {eff }}=L+8 /(3 \pi) D \text {, }
$$

where $L$ is the orifice length (thickness of the cavity top wall). Besides, Kinsler [26] suggests a bit shorter $L_{\text {eff }}$ for a circular hole in a thin wall of a resonator:

$$
L_{\text {eff }}=L+0.8 D \text {. }
$$

Figure 3 shows that the present data reasonably agree with Eq. (1) with the $L_{\text {eff }}$ from Eq. (2). The maximum deviation between the present data and values predicted by Eqs. ( 1 and 2) is less than $10 \%$ in the whole investigated range. Moreover, using Eq. (3) decreases this deviation for a majority of experimental data points: the maximum deviations between the present data and values predicted by Eqs. (1 and 3) are within $7 \%$ for $h=10-30 \mathrm{~mm}$.

These measurements indicate that the tested cavity resonates as the Helmholtz resonator. The results 
demonstrate possibility of the experiments and validate the present approach.

\subsection{Aeroacoustics of the pipe-cavity-orifice nozzle}

Acoustic effects of the pipe-cavity-orifice nozzle on the jet flows was evaluated by means of the power spectral density (PSD). Investigated ranges were $R e=1600$ 18000 and $h=7-40 \mathrm{~mm}$. Figures 4-7 present typical results plotted as the relative amplitude of the PSD for jets without passive control (i.e. $h=0$ ) and jets under the cavity control effect at $h=7-20 \mathrm{~mm}$.

Figure 4 shows that the cavity control effect for $R e=1600$ is relatively weak. Despite some redistribution in PSD can be identified, at $h=8$ and $10 \mathrm{~mm}$, no significant increase of sound pressure level (SPL) was found. In fact, for all cases at $R e=1600$ (figure 4), the SPL was evaluated below a resolution of the instruments, i.e. below $40 \mathrm{~dB}$.

Table 1 summarizes SPL measurements for all investigated jets at $R e=1600-18000$. The maximum obtained SPL for various $R e$ by means of the cavity control effect is named $\mathrm{SPL}_{\max }$. Table 1 shows that an increase of the $R e$ causes stronger sound generation. The highest SPL in this study was $\mathrm{SPL}_{\max }=79.3 \mathrm{~dB}$; it was achieved at $\operatorname{Re}=18000$ and $h=7 \mathrm{~mm}$.

Table 1. SPL of jets without passive control $(h=0)$ and jets under the cavity control effect at $h=7-15 \mathrm{~mm}$.

\begin{tabular}{cccc}
\hline & $\begin{array}{c}\text { No control } \\
(h=0) \\
\mathrm{SPL}\end{array}$ & \multicolumn{2}{c}{ With control } \\
$\operatorname{Re}$ & $\begin{array}{c}\text { SPL } \max \\
(\mathrm{dB})\end{array}$ & $h(\mathrm{~mm})$ \\
\hline \hline 1600 & $<40$ & $<40$ & 8 \\
4400 & 41.2 & 50.8 & 15 \\
8300 & 49.7 & 72.8 & 10 \\
18000 & 55.4 & 79.3 & 7 \\
\hline
\end{tabular}

As is indicated by figures 5-7, distinct peaks in PSD appeared as results of the passive control at $R e=4400$ 18000 . This effect indicates significant changes in jet structures. The level of the PSD peaks is markedly higher than PSD of the uncontrolled reference jets, namely by 20 $\mathrm{dB}$ at $R e=4400$ and even by $50 \mathrm{~dB}$ at $R e=8300$ and 18000 . These peaks indicate a resonant behavior of the whole system. It can be deduced that the present nozzle design can be useful for a passive control of jet flows.

Moreover, a distinguishable cascade of peaks with lower PSD level can be identified in figures 5(b), 6(b), and $7(b)$, which resembles higher harmonics of the Helmholtz resonator. However, the aeroacoustic behavior of the present system with fluid flow is more complicate than the Helmholtz resonator. As was discussed by Henrywood and Agarwal [23], the excitation mechanism of a such system consists of a many particular sources such as (1) resonance behavior of the supplied piping system, (2) vortex shedding at the sudden expansion of the cross-sectional area (pipe-cavity interface), (3) cavity operating as the Helmholtz resonator, (4) sound propagation via edge tones at the jet impingement inside the cavity (cavity-orifice interface), (5) vortex shedding in the jet shear layer, and (6) turbulent noise of the jets inside as well as outside the cavity.

\subsection{Flow visualization}

Because the aeroacoustic effects described above are the most intensive at $R e=18000$, flow visualization experiments and velocity measurements focus on this $R e$ case, when the control effects can be expected well distinguishable.
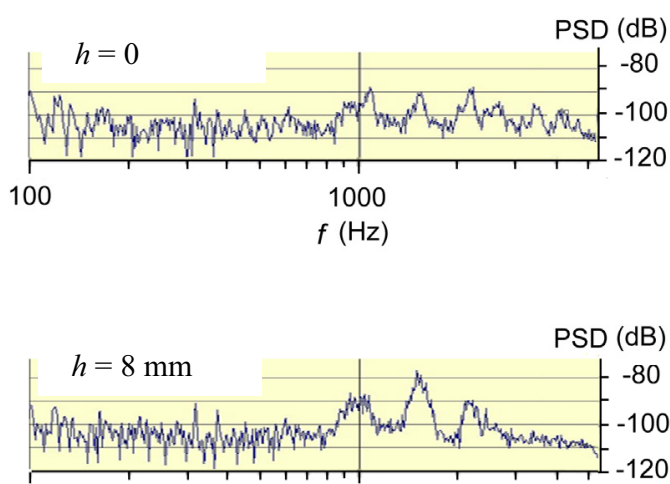

(b)

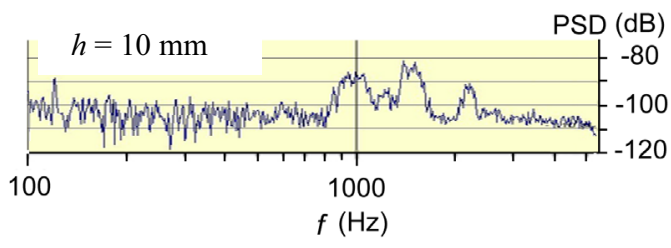

Figure 4. Power spectral density for $R e=1600$, (a) jet without a passive control, (b) jet under the cavity control effect at $h=8 \mathrm{~mm}$ and $10 \mathrm{~mm}$.
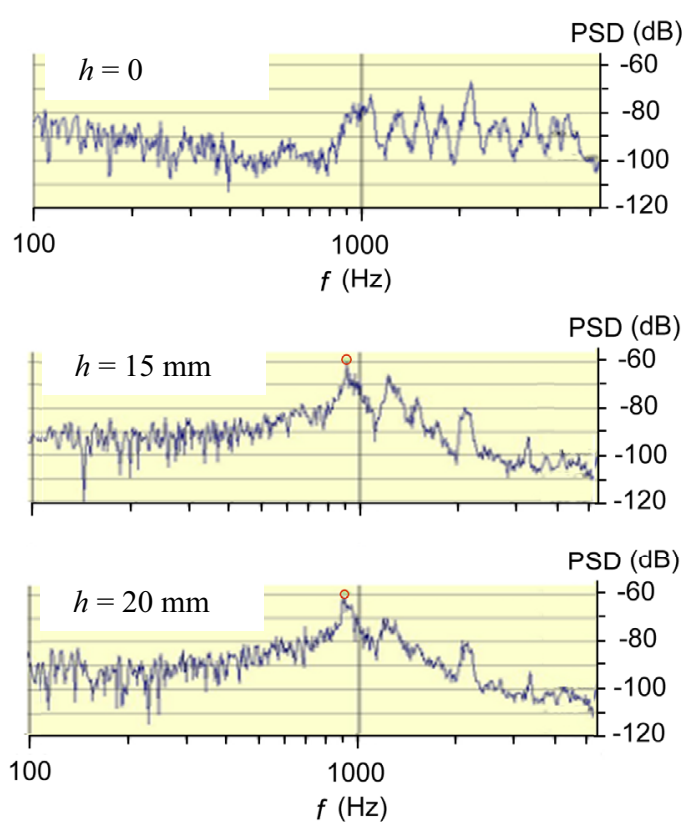

Figure 5. Power spectral density for $R e=4400$, (a) jet without a passive control, (b) jet under the cavity control effect at $h=15 \mathrm{~mm}$ and $20 \mathrm{~mm}$. 


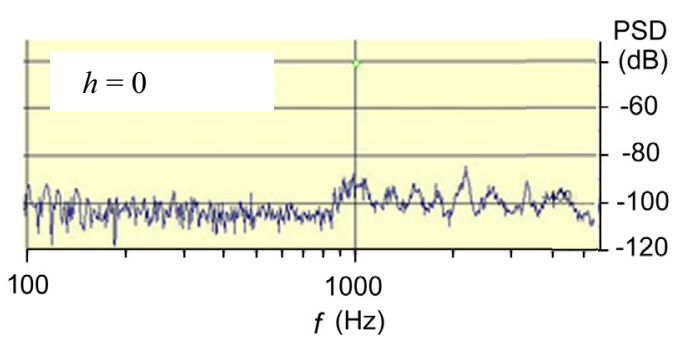

(a)
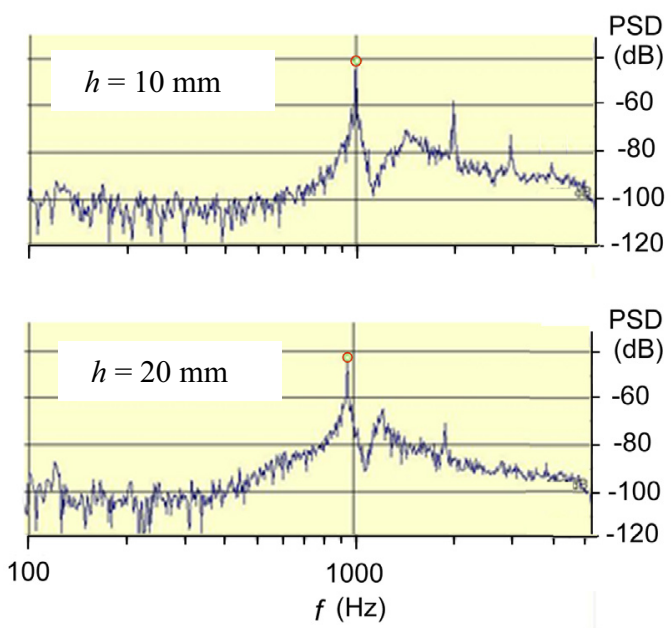

(b)

Figure 6. Power spectral density for $R e=8300$, (a) jet without a passive control, (b) jet under the cavity control effect at $h=10 \mathrm{~mm}$ and $20 \mathrm{~mm}$.

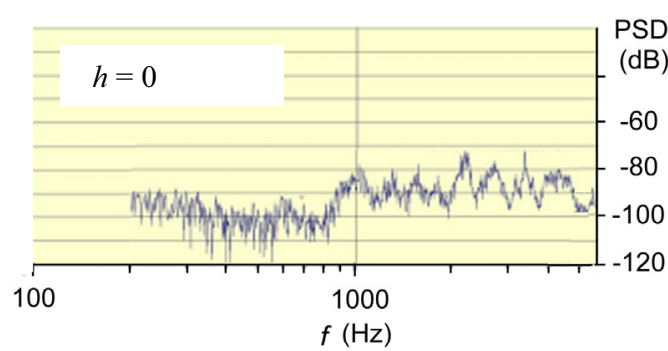

(a)

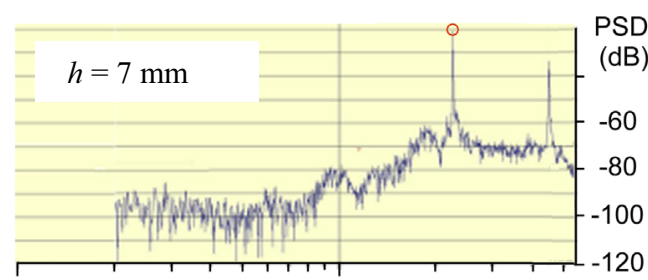

(b)

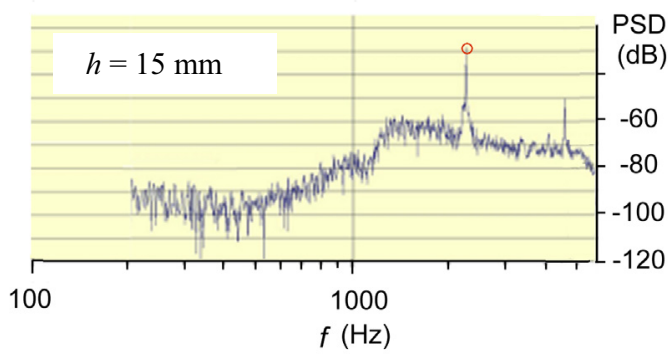

Figure 7. Power spectral density for $R e=18000$, (a) jet without a passive control, (b) jet under the cavity control effect at $h=7 \mathrm{~mm}$ and $15 \mathrm{~mm}$.

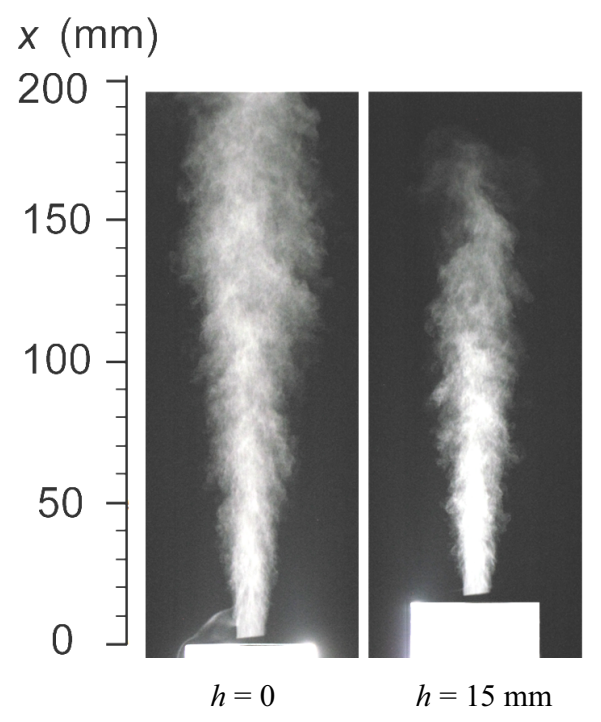

(a)

(b)

Figure 8. Flow visualization for $R e=18000$; (a) without passive control $(h=0)$, (b) under the cavity control effect at $h=15 \mathrm{~mm}$.

Figures $8(\mathrm{a}, \mathrm{b})$ show typical flow visualization for $R e=18000$, for the jet without a passive control (ie. at $h=0$, see figure $8(\mathrm{a})$ ) and for the jet under the cavity control effect at $h=15 \mathrm{~mm}$, see figure 8(b). The control effect can be identified as a slight spreading and shortening of the visible jet flow area. However, flow visualization cannot capture unambiguously this effect. It will be quantified by the time-mean velocity measurement in the following text.

\subsection{Velocity measurement of the flow field}

Figure 9(a) shows the streamwise variation of the timemean velocity along the jet centerline for $R e=18000$ at various cavity depth from $h=0$ (i.e. without a passive control) to $h=20 \mathrm{~mm}$. A nondimensionalization of the velocity is made by the centerline velocity in the orifice $u_{0}$.

A behavior of a jet velocity decay is well comparable for all investigated jets. Firstly, the centerline jet velocity is approximately constant up to $x / D=4$, i.e. the measurement is made in the jet core of in the initial region. Further downstream, the velocity decreases (the transition region) towards the final inverse proportionality of the velocity decay (fully developed region of the turbulent jet): $u / u_{0} \sim(x / D)^{p}$, where the exponent $p$ is approximately -1 . This development is well-known from literature for fully developed axisymmetric turbulent jets - see Schlichting and Gersten [1] and Blevins [27]. A good agreement with literature is demonstrated by the relationship $u / u_{0}=6 /(x / D)$ by Blevins [27], which is plotted as the dashed line in figure 9(a) too.

The control effect is well manifested by a shortening of the transition region and a sooner formation of the final inverse proportionality of the velocity decay indicating the fully developed region - the control effect is depicted by the arrow in figure 9(a). Note that the 

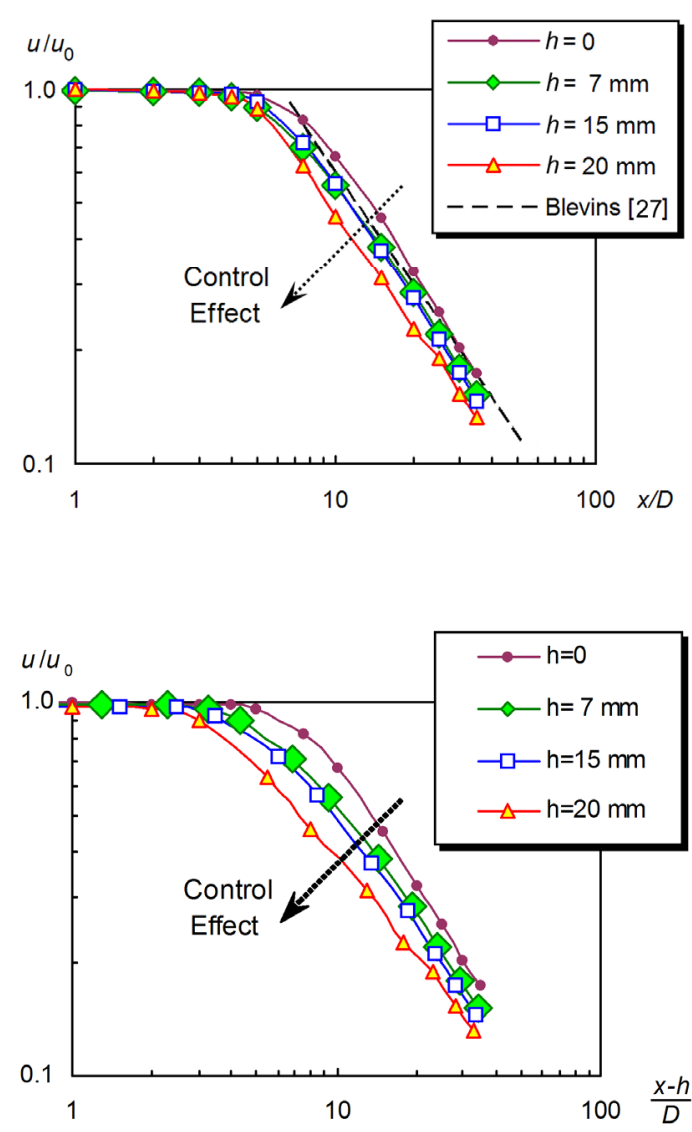

Figure 9. The centerline velocity decay at $R e=18000$ as a function of axial distance.

exponent $p=-1$ of the exponential velocity decay was not changed by the control effect - see figure 9 too.

It is worthy of notice here that the streamwise coordinate $x / D$ in figure 9 (a) was purposely chosen in agreement with the paper by Hill and Greene [8]. On the other hand, another logical streamwise coordinate can originate in the outlet orifice cross section, thus the data presentation from figure 9(a) can be transformed into figure 9 (b) with $(x-h) / D$ horizontal coordinate. Obviously, all the above described results demonstrating the control effect in figure 9(a) are evident in figure 9(b) too - in fact, the control effect is manifested in figure 9 (b) even more clearly.

The present paper uses an axially symmetric setup thus we can expect an axially symmetric jet flows. Nevertheless, the expected symmetry of the jets has to be experimentally verified to exclude influences of manufacturing inaccuracies or random disturbances or suchlike. Figure 10 shows three typical velocity profiles at approximately $R e=18000$, which were measure for $h$ $=0$ (i.e. without a passive control) at $x=10,100$, and $200 \mathrm{~mm}$. The profiles demonstrate the jet flow development, i.e. and decreasing of the centerline velocity and widening of the jet flow. Figure 10 demonstrates an acceptable symmetry of the velocity profiles. Obtained velocity profiles consisting of two halves for $r \leq 0$ and $r \geq 0$ were transformed into one symmetrical profile $u(r)$ by averaging the $u(+r)$ data and $u(-r)$ data for each data point of the traversing line. Figure 10 shows experimental data as well as the

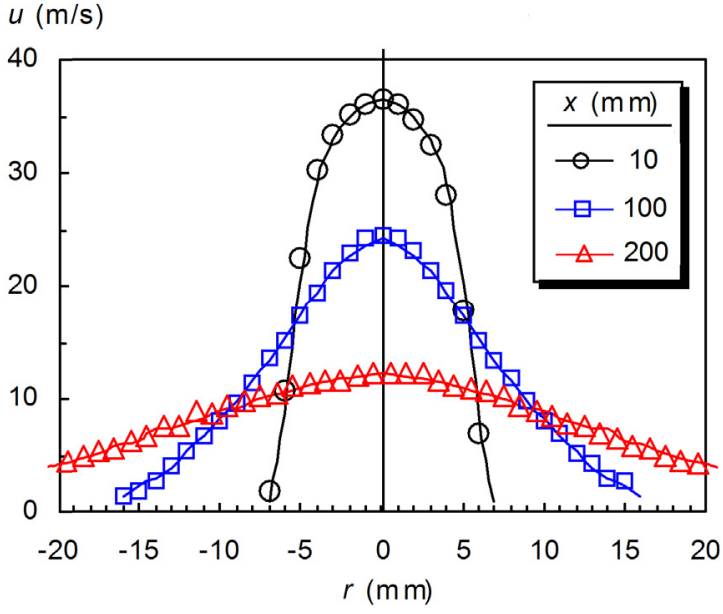

Figure 10. Velocity profiles at $h=0$ : points present experimental data, lines are symmetrical profiles obtained by the data symmetrization process.
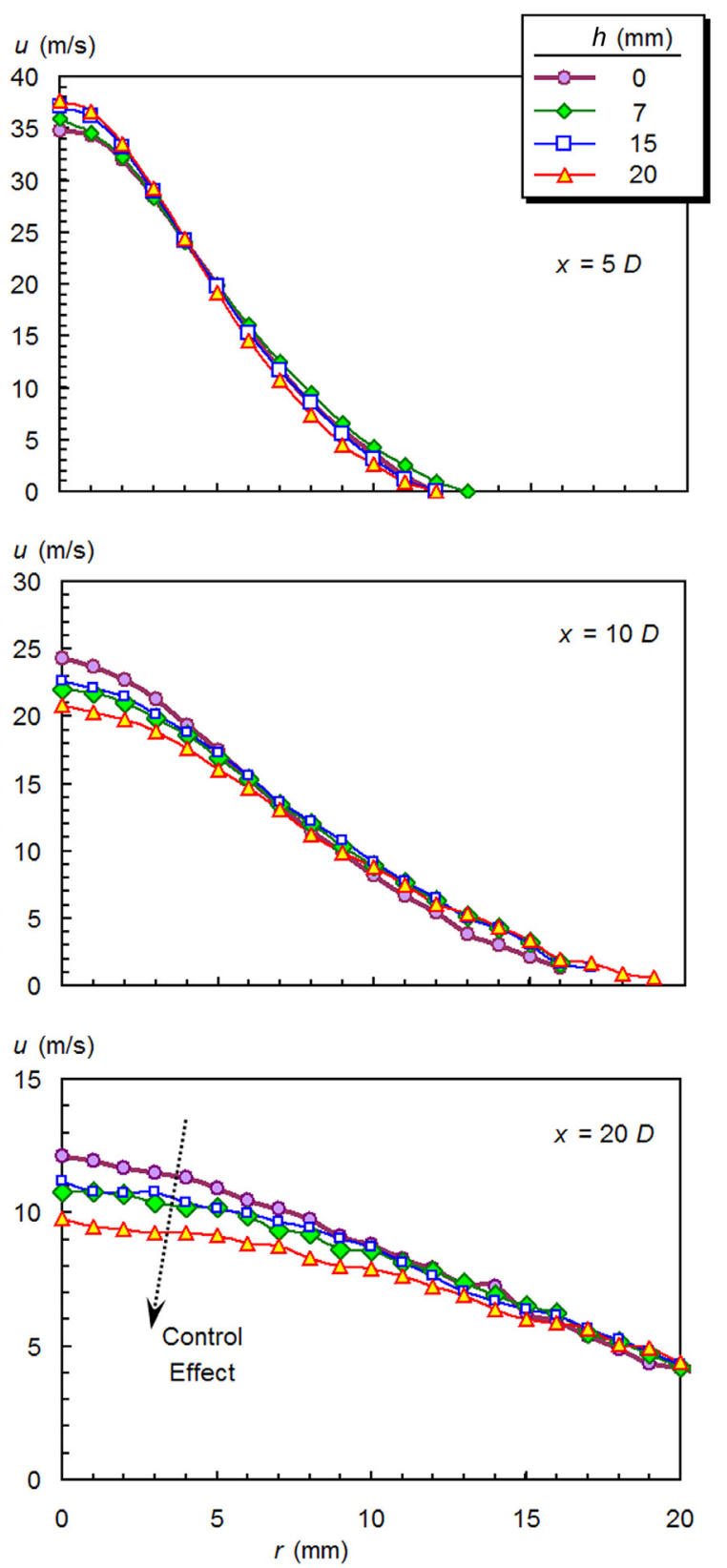

Figure 11. Velocity profiles at $R e=18000$. 
symmetrical profiles resulting from the described data symmetrization process.

Figure 11 demonstrates a flow development for jets without/with the flow control: the centerline velocity decreases with increasing $x$ and the jets are widened at the same time. The present control effect is well manifested by decreasing velocities in the central part, as is depicted by the arrow in figure 11 at $x=20 \mathrm{D}$.

To compare jet flow development with available literature, the jet half-width was evaluated from experimental velocity profiles. The jet half-width (or half-radius for the present axisymmetric case) is the transverse distance from the axis to the point in which the axial velocity $u$ falls to the one-half of the centerline value $u_{\max }$. Figure 12 presents a development of the jet half-width for $R e=18000$ and a comparison with a formula by Schlichting and Gersten [1], $r_{0.5} / D=0.09(x / D+1)$, which can be illustrated as the apical angle of $10.3^{\circ}$ with the apex in the virtual origin of the jet at $x=-D$. Another relevant formula is $r_{0.5} / D=0.086 x / D$ with the apical angle of $9.8^{\circ}$, as proposed Blevins [27]. The both reference formulae are plotted in figure 12 too and an acceptable agreement of the present data with these references can be concluded.

Figure 13 shows the velocity profiles after transformation into a dimensionless form of $u / u_{\max }\left(r / r_{0.5}\right)$. Figure 13 demonstrates a similarity of the investigated profiles resulting from fully developed states of the jets, because they collapse quite well into one nondimensional profile. For comparison purposes, figure 13 shows also the reference profile by Blevins [27]: $u / u_{\max }=\mathrm{e}^{-94\left(0.086 r / r_{0.5}\right)^{2}}$. A quite good agreement of the present data with this formula [27] can be concluded (except a very few points at the jet peripheral parts for $x=$ $20 D$, where a measurement of a small velocity $u<2 \mathrm{~m} / \mathrm{s}$ is problematic).

\subsection{Integral results}

The velocity profiles can be integrated to obtain following integral quantities, namely the volume flow

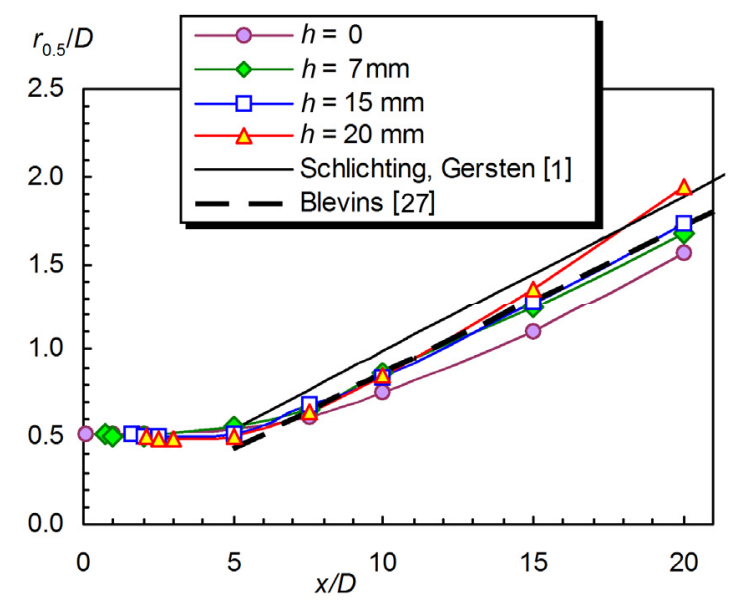

Figure 12. Development of the half-width of the jets at $R e=18000$. rate $Q$, the flux of the axial momentum $M$, and the kinetic energy flux $E$. The evaluations are defined as $Q=2 \pi \int_{0}^{+\infty} u r \mathrm{~d} r, M=2 \pi \rho \int_{0}^{+\infty} u^{2} r \mathrm{~d} r$, and $E=\pi \rho \int_{0}^{+\infty} u^{3} r \mathrm{~d} r$.

These integrations were applied with finite integration upper limits on the present experimental data for various cross sections from $x=0$ to $x=20 \mathrm{D}$. The obtained results are presented in figure 14 in dimensionless forms of $Q / Q_{0}, M / M_{0}$, and $E / E_{0}$, where $Q_{0}, M_{0}$, and $E_{0}$ are the initial quantities evaluated at the nozzle.

Figure 14(a) shows a development of the volume flow rate along the jet axis, which increases because of fluid entrainments from surroundings. Figure 14(b) shows that the momentum flux along the jet axis slightly decreases, and this decrease is promoted by the control effect - see the arrow in figure 14(b) and $29 \%$ decrease at $x / D=20$. Finally, the kinetic energy flux along the jet axis significantly decreases (see figure $14(\mathrm{c})$ ) and the control
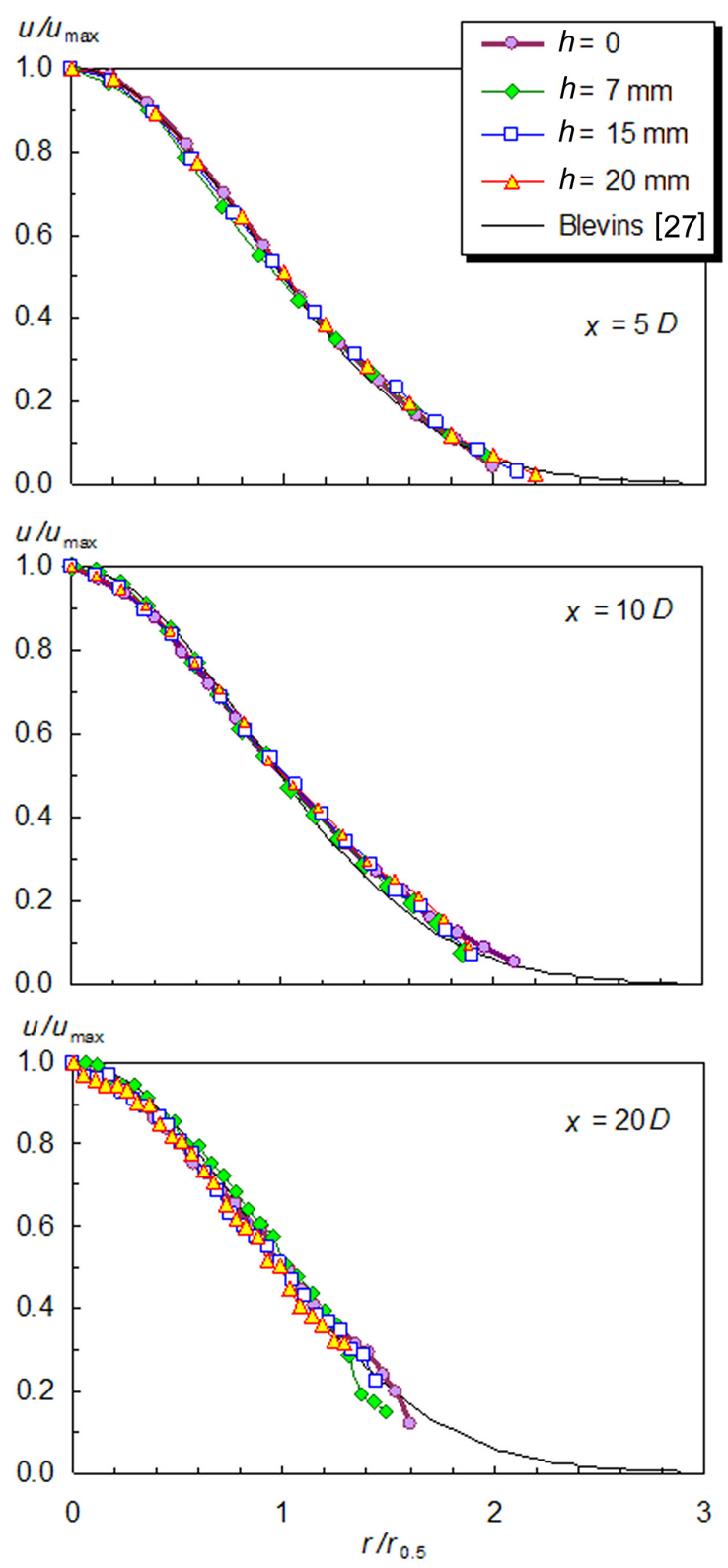

Figure 13. Similarity of dimensionless velocity profiles at $\operatorname{Re}=18000$. 
effect intensifies this process - see the arrow in figure 14 (c) and $46 \%$ decrease at $x / D=20$.

To assess these results, a comparison with available literature was performed. The volume flow rate increase resulting from the fluid entrainment can be expressed by the following formula (Blevins [27]):

$$
Q / Q_{0}=0.32 x / D .
$$

The momentum flux along the jet axis is very nearly constant, as was approximated by Schlichting and Gersten [1], and Blevins [27]. More realistic results were derived theoretically and confirmed experimentally by Kotsovinos [28] who described a small decrease of the momentum flux by the following formula:

$$
M / M_{0}=0.983-0.0693 \ln [x /(6 D)] .
$$
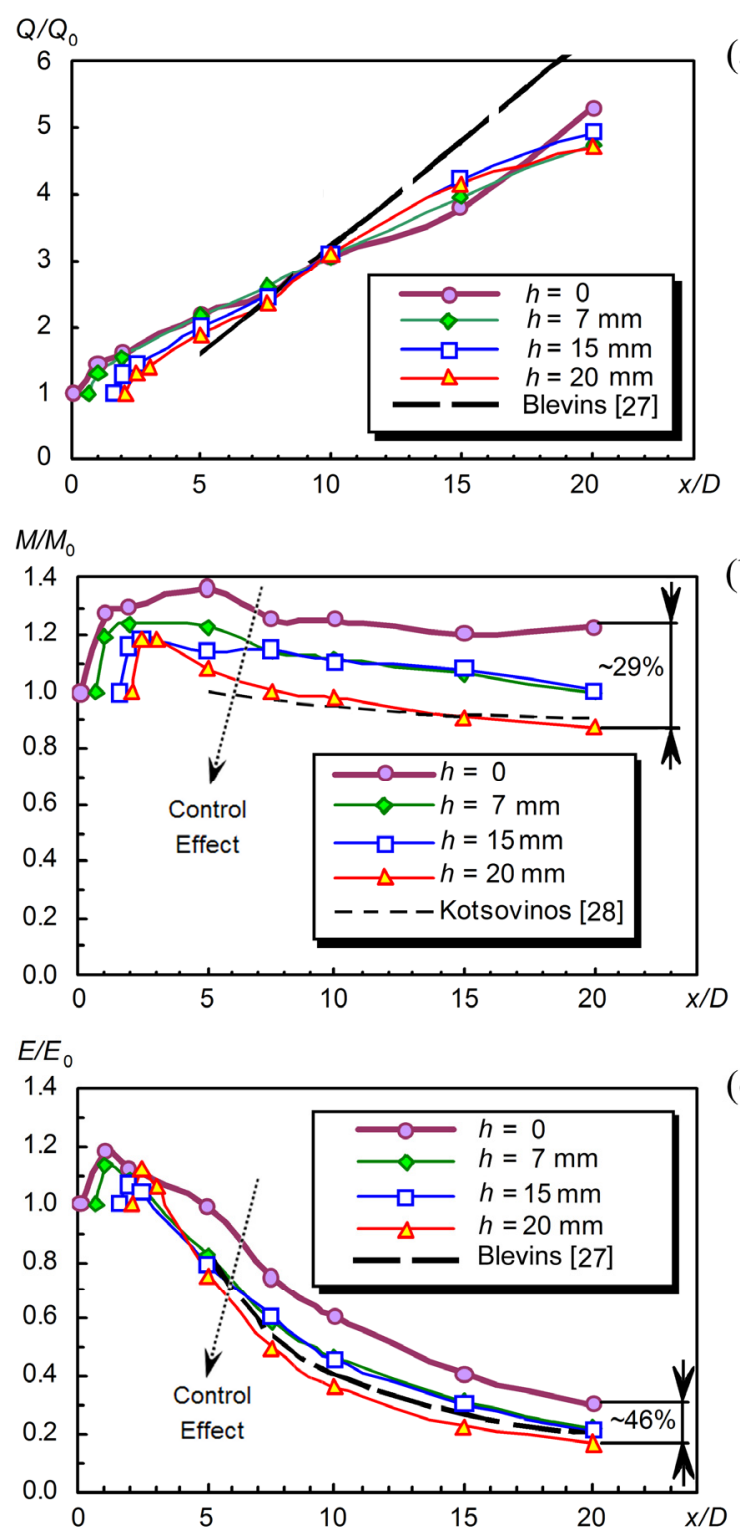

Figure 14. Integral characteristics of jets at $R e=18000$; (a) volume flow rate, (b) momentum flux, and (c) kinetic energy flux.
Finally, the kinetic energy flux along the jet axis decreases as is described by the formula by Blevins [27]:

$$
E / E_{0}=4.1 D / x .
$$

Figure 14 shows a reasonable agreement of the present results with references [1, 27, 28]. Systematic shifts of the curves in figures 14(b and c), which is depicted by the arrows, indicate the revealed control effect. Namely, the fluid mixing is enhanced and velocity decay along the axis is promoted, hence the momentum and kinetic energy fluxes decrease more intensively in the controlled jets in comparison with common jets. The decreases by $29 \%$ and $46 \%$ are depicted in figures 14 too.

\section{Conclusions}

A pipe-cavity-orifice nozzle was designed. The nozzle consists of a pipe of the inner diameter of $10 \mathrm{~mm}$ and the length of 15 diameters, which is equipped with an axisymmetric cavity with an adjustable depth at the pipe end.

Firstly, the cavity resonance behavior was tested without jet flows. Frequencies of the sound effects were evaluated by means of a microphone measurement. A signal on-line analysis was made to obtain the power spectral density (PSD). The evaluated resonant frequencies well agree with a theoretical prediction of the natural frequency of the Helmholtz resonator. The maximum deviation between the present data and the theory is less than $7-10 \%$.

The nozzle was used for generation of axisymmetric air jet flows with a passive flow control by means of selfsustained acoustic excitations. The jets were experimentally investigated using flow visualization, microphone measurements and time-mean velocity measurement by the Pitot probe. The PSD and the sound pressure level (SPL) were evaluated from microphone measurements. The jet Reynolds number ranged $R e=$ 1600-18 000. The most effective aeroacoustics response was found at $R e=18000$, therefore a majority of experiments was made at this $R e$ regime.

The results demonstrate effects of the passive control on the jet behavior. In comparison with common jets, fluid mixing is enhanced and velocity decay along the axis is promoted by a shortening of the jet transition region. On the other hand, an inverse proportionality of the velocity decay $(u \sim 1 / x)$ in the fully developed region is not changed.

The control effect was also indicated from the integral quantities of the jet. The flux of the axial momentum and the kinetic energy flux decrease more intensively in comparison with common jets.

\section{Acknowledgments}

The authors gratefully acknowledge the support from the Grant Agency CR - the Czech Science Foundation (Project No. 14-08888S) and the institutional support RVO:61388998. 


\section{References}

1. H. Schlichting, K. Gersten K. Boundary-Layer Theory (Springer-Verlag, Berlin, 2000)

2. G.N. Abramovich, The Theory of Turbulent Jets (MIT Press, Massachusetts, 1963) (original in Russian: Teoriia turbulentnykh strui, Moscow, GIFML, 1960).

3. S.C. Crow, F.H. Champagne, J. Fluid Mech. 48 (1971)

4. Mohamed Gad-el-Hak, Flow Control: Passive, Active, and Reactive Flow Management (Cambridge University Press, Cambridge, New York, 2007)

5. F.O. Thomas, Appl. Mech. Rev. 44 (1991)

6. Vlasov E.V., Ginevskii A.S., Sov. Phys. Acoust. 26, 1 (1980)

7. T. Liu, J. P. Sullivan, Int. J. Heat Mass Transfer 39, 17 (1996)

8. W. G. Hill Jr., P. R. Greene, Trans. ASME J. Fluids Eng. 99 (1977)

9. M. A. Z. Hasan and A. K. M. F. Hussain, J. Fluid Mech. 115 (1982)

10. A. K. M. F. Hussain and M. A. Z. Hasan, J. Fluid Mech. 134 (1983)

11. C.J. Abell, R. E. Luxton, Mixing enhancement in combusting flow (Technical Report 1979-1981, The University of Adelaide, Department of Mechanical Engineering, Adelaide, Australia) (cited by Lee [12])

12. S.-K. Lee, Study of a naturally oscillating triangular-jet flow (PhD. Thesis., University of Adelaide, School of Mech. Eng., 2009)

13. G.J. Nathan, The enhanced mixing burner (PhD. Thesis., University of Adelaide, Dept. Mech. Eng., 1988)

14. R.E. Luxton, G.J. Nathan, Controlling the motion of a fluid jet (Patent US 5060867 A, 1991)

15. G.J. Nathan, S.J. Hill, R.E. Luxton, J. Fluid Mech. 370 (1998)

16. R.H. Page, P.S. Chinnock, J. Seyed-Yagoobi, AIAA J. of Thermophysics and Heat Transfer 10, 2 (1996)

17. H. Viets, AIAA J. 13, 10 (1975)

18. G.Raman, E.J.Rice, D.M.Cornelius, Trans. ASME, Jour. Fluids Eng. 116 (1994)

19. C. Camci, F. Herr, Trans. ASME Jour. Heat Transfer 124 (2002)

20. A. Seifert, S. Pastuer, Method and mechanism for producing suction and periodic excitation flow (US Patent No. 7,055,541 B2, 2006)

21. V. Tesař, Z. Trávníček, J. Kordík, Z. Randa, Sens. Actuator A-Phys. 138, 1 (2007)

22. J. Mi, G.J. Nathan, R.E. Luxton, Flow Turbul. Combust. 67 (2001)

23. R. H. Henrywood, A. Agarwal, Phys. Fluids 25 (2013)

24. P. Pušková, Passive control of axisymmetric air flow (Pasivní ř́zení osově symetrického proudu vzduchu) (Master thesis, Czech Technical University, Faculty of Mechanical Engineering, Prague, Czech Republic, 2015) (in Czech)

25. S. J. Kline, F.A. McClintock, Mechanical Engineering 75 (1953)
26. L.E. Kinsler, A.R. Frey, A.B. Coppens, J.V. Sanders, Fundamentals of Acoustic (Wiley, New York, 2000)

27. R. D. Blevins, Applied Fluid Dynamics Handbook (Florida: Krieger Publishing Company, 2003)

28. N.E. Kotsovinos, J. Fluid Mech. 87, 1 (1978) 